\title{
Chronic Unilateral Uveitis with Macular Edema Secondary to Dabrafenib for Pilocytic Astrocytoma
}

\author{
Matthew Chang $^{a}$ Robin K. Kuriakose ${ }^{b}$ Kunyong $\mathrm{Xu}^{\mathrm{c}}$ \\ David R.P. Almeidad Eric K. Chin ${ }^{b}, e, f$ \\ aLoma Linda University School of Medicine, Loma Linda, CA, USA; b Loma Linda University \\ Eye Institute, Loma Linda, CA, USA; 'Department of Ophthalmology and Visual Sciences, \\ University of Arizona, Tucson, AZ, USA; ${ }^{\mathrm{d} E r i e}$ Retinal Surgery, Erie, PA, USA; ${ }^{\mathrm{e}}$ Retina \\ Consultants of Southern California, Redlands, CA, USA; ' ${ }^{\prime}$ oma Linda University Medical \\ Center, Veterans Affair Hospital, Loma Linda, CA, USA
}

\section{Keywords}

Uveitis · Macular edema · Dabrafenib · Pilocytic astrocytoma

\begin{abstract}
This report describes a unique case of chronic unilateral anterior uveitis associated with macular edema while on oral dabrafenib treatment for chronic recurrent pilocytic astrocytoma. After gradual taper of prednisolone acetate OS, the patient developed recurrent mild lowgrade anterior uveitis and macular edema OS that required low dose of prednisolone acetate OS to prevent recurrences while on oral dabrafenib. When oral dabrafenib was temporarily discontinued for 3 months due to her ocular inflammation, she had no flares of her uveitis; however, her tumor increased significantly in size. The collaborative decision was made to continue her oral dabrafenib while on topical anti-inflammatory therapy for her uveitis. Clinicians should be aware of this potential unilateral sequela of uveitis secondary to dabrafenib. Further investigation should be conducted to identify factors that may place certain patients at higher risk for this complication.
\end{abstract}

\section{Introduction}

Pilocytic astrocytoma arises in the pediatric population and is typically benign. The 10 -year survival rate is $>90 \%$ in children but has a higher mortality in adults $[1,2]$. Approximately $6-10 \%$ of pilocytic astrocytomas develop activating mutations in the BRAF V600E 
Chang et al.: Chronic Uveitis with Macular Edema due to Dabrafenib for Pilocytic Astrocytoma

oncogene which leads to the transcription of genes that increase the proliferation of tumor cells [3].

Dabrafenib (Tafinlar) was approved in 2013 for the treatment of metastatic melanoma. It is an oral antitumor drug that selectively inhibits the BRAF V600E kinase, the product of the BRAF V600E oncogene, thereby inhibiting the growth of tumor cells. Ocular side effects of dabrafenib have been reported in patients with metastatic melanoma and may include anterior and posterior uveitis, bilateral panuveitis, papillitis, and retinal detachment [3]. We report a unique case of chronic unilateral anterior uveitis associated with macular edema while on oral dabrafenib treatment for chronic recurrent pilocytic astrocytoma.

\section{Case Report}

A woman in her late 30s with history of a pilocytic astrocytoma treated with surgical resection 8 years prior, presented with blurred vision OS, with a best-corrected visual acuity of 20/40 OD and 20/70 OS. Her intraocular pressures were normal OU. There were no remarkable findings OD except mild cataract. Slit lamp examination OS demonstrated 1+ cell/ flare with patchy posterior synechiae inferonasally and inferotemporally without pupillary block, and a mild cataract. Fundus examination OS demonstrated 1+ anterior vitreous cell and macular edema (Fig. 1). She was started on topical cyclopentolate TID and topical prednisolone acetate QID OS, with gradual resolution of her inflammation and macular edema. After gradual taper of these drops, she developed recurrent mild low-grade anterior uveitis and macular edema and has required low dose of prednisolone acetate BID OS to prevent recurrences while on oral dabrafenib.

She had been on oral dabrafenib since her surgery 8 years prior. She had no history of prior radiation or other chemotherapy. When oral dabrafenib was temporarily discontinued for 3 months due to her ocular inflammation, she had no flares of her uveitis; however, her tumor increased significantly in size. The collaborative decision was made to continue her oral dabrafenib while on topical anti-inflammatory therapy for her uveitis.

\section{Discussion}

Ophthalmic side effects of dabrafenib began after the drug was FDA-approved for metastatic melanoma in 2013 and has been associated with uveitis at a rate of $1-10 \%$ [4, 5]. While symmetrical uveitis sequelae is more common, asymmetric unilateral presentations may also
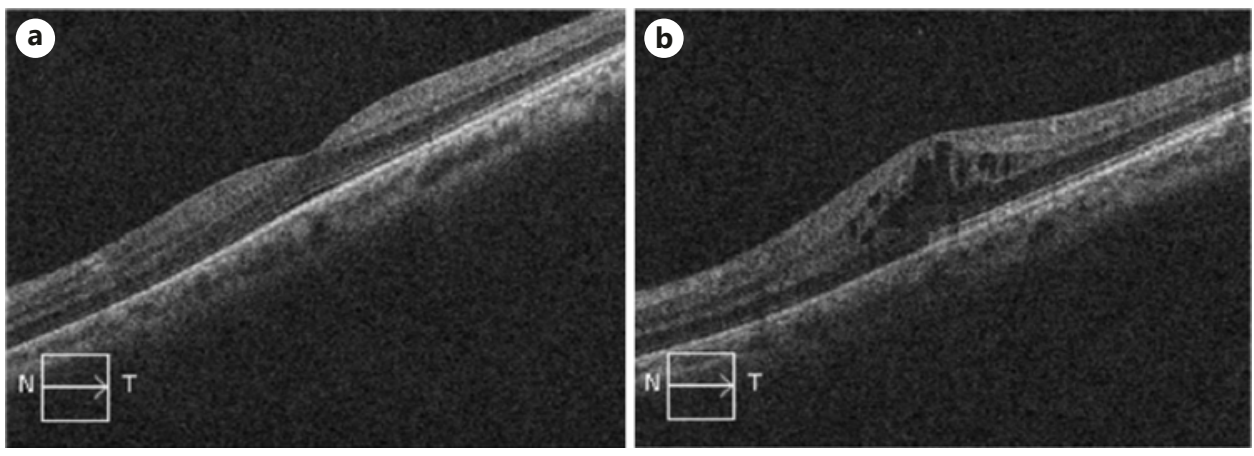

Fig. 1. OCT of the macula demonstrating no central macular edema in the right eye (a) and unilateral left eye edema while on dabrafenib therapy (b). OCT, optical coherence tomography. 
Chang et al.: Chronic Uveitis with Macular Edema due to Dabrafenib for Pilocytic Astrocytoma

be seen as in our case [6]. In this report, we highlight the first case of a patient developing chronic unilateral anterior uveitis in response to dabrafenib therapy for the treatment of pilocytic astrocytoma.

Based on handful of case reports found in the literature, it appears the time from initiation of dabrafenib and presentation with uveitis ranges from week to months, although there has been a report of uveitis arising in a matter of days, as well as a report of uveitis presenting 4 years later. To our knowledge, this is the first report of uveitis arising 8 years after initiation of oral dabrafenib [7-10].

Besides dabrafenib, several other drugs that target the MAPK pathway have been associated with uveitis $[6,11,12]$. Due to very similar mechanisms (Fig. 2), uveitis has also been reported with vemurafenib, encorafenib plus binimetinib, and trametinib plus cobimetinib use $[6,11,12]$. Inhibition of epidermal growth factor receptor (EGFR), an upstream mediator of the MAPK pathway, via erlotinib or gefitinib has been associated with uveitis $[5,13]$. It has been hypothesized that dabrafenib induces uveitis through breakdown of the blood-retinal barrier, a possible effect of MAP kinase inhibition, eventually leading to compromise of ocular immune privilege and autoimmune uveitis [14].

Clinicians should be aware of this potential unilateral sequela of dabrafenib in treatment of primary tumors such as pilocytic astrocytomas. Further investigation should be conducted to identify factors that may place certain patients at higher risk for this complication. Patients should be monitored and maintenance topical corticosteroids may be used to prevent recurrences.

Fig. 2. Schematic of the MAPK kinase pathway demonstrating cellular targets of various drugs.

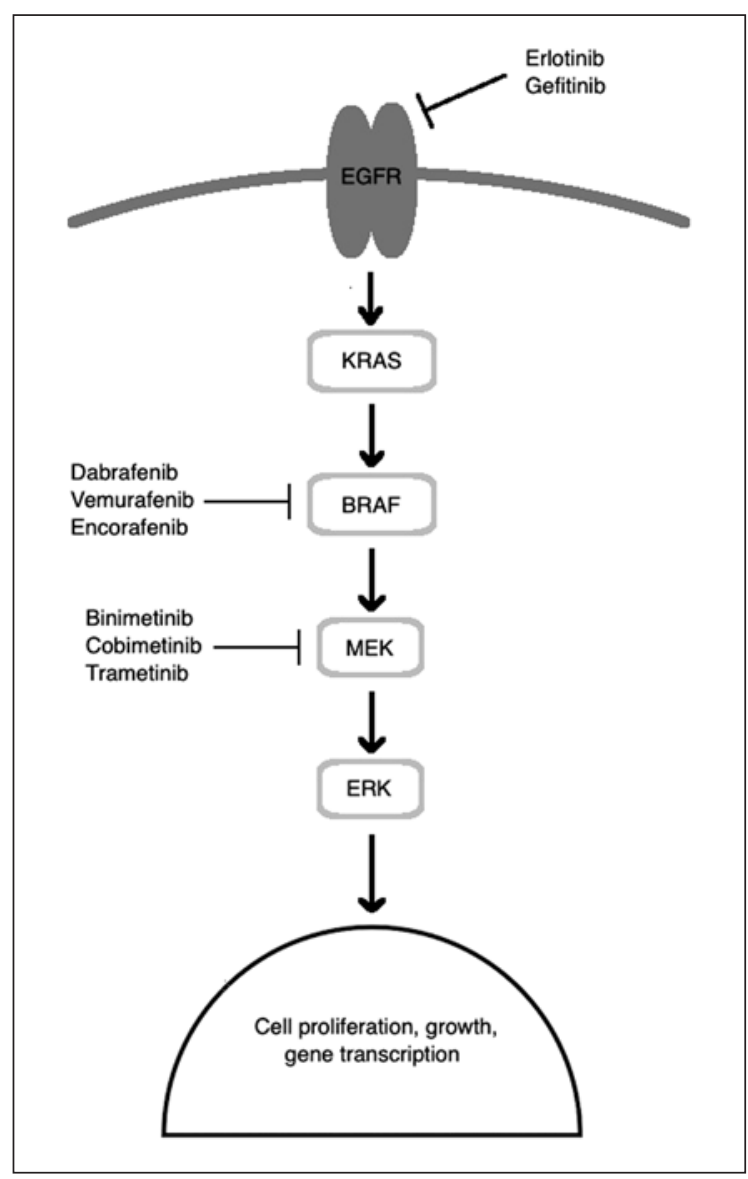


Chang et al.: Chronic Uveitis with Macular Edema due to Dabrafenib for Pilocytic Astrocytoma

\section{Statement of Ethics}

Subject has given their written informed consent to publish their case (including publication of images). Full adherence to the Declaration of Helsinki and all federal and state laws.

\section{Conflict of Interest Statement}

Financial \& Proprietary Disclosure: Almeida: Allergan (Honoraria), Alcon (Honoraria), Genentech (Honoraria), and Novartis (Honoraria).

\section{Funding Sources}

No funding sources to disclose.

\section{Author Contributions}

All authors listed above meet the criteria for ICMJE authorship, including substantial contributions to the conception or design of the work; the acquisition, analysis, or interpretation of data for the work, drafting the work, or revising it critically for important intellectual content; and final approval of the version to be published and agreement to be accountable for all aspects of the work in ensuring that questions related to the accuracy or integrity of any part of the work are appropriately investigated and resolved.

\section{References}

1 Bornhorst M, Frappaz D, Packer RJ. Pilocytic astrocytomas. Handb Clin Neurol. 2016;134:329-44.

2 Johnson DR, Brown PD, Galanis E, Hammack JE. Pilocytic astrocytoma survival in adults: analysis of the surveillance, epidemiology, and end results program of the National Cancer Institute. J Neurooncol. 2012;108(1): 187-93.

3 Olow A, Mueller S, Yang X, Hashizume R, Meyerowitz J, Weiss W, et al. BRAF status in personalizing treatment approaches for pediatric gliomas. Clin Cancer Res. 2016;22(21):5312-21.

4 Ballantyne AD, Garnock-Jones KP. Dabrafenib: first global approval. Drugs. 2013;73(12):1367-76.

5 Huillard O, Bakalian S, Levy C, Desjardins L, Lumbroso-Le Rouic L, Pop S, et al. Ocular adverse events of molecularly targeted agents approved in solid tumours: a systematic review. Eur J Cancer. 2014;50(3):638-48.

6 Gavric AU, Ocvirk J, Mekjavic PJ. Ocular changes in metastatic melanoma patients treated with MEK inhibitor cobimetinib and BRAF inhibitor vemurafenib. Radiol Oncol. 2018;52(2):213-9.

7 Taylor SC, Hrisomalos F, Linette GP, Rao PK. A case of recurrent bilateral uveitis independently associated with dabrafenib and pembrolizumab therapy. Am J Ophthalmol Case Rep. 2016;2:23-5.

8 Woltsche N, Kruger MA, Weger M, Wolf IH, Seidel G. Intravitreal steroid treatment for uveitis associated with dabrafenib and trametinib for metastatic cutaneous melanoma. Ocul Immunol Inflamm. 2020;1-3.

9 Lim J, Lomax AJ, McNeil C, Harrisberg B. Uveitis and papillitis in the setting of dabrafenib and trametinib therapy for metastatic melanoma: a case report. Ocul Immunol Inflamm. 2018;26(4):628-31.

10 Draganova D, Kerger J, Caspers L, Willermain F. Severe bilateral panuveitis during melanoma treatment by Dabrafenib and Trametinib. J Ophthalmic Inflamm Infect. 2015;5:17.

11 Dummer R, Ascierto PA, Gogas HJ, Arance A, Mandala M, Liszkay G, et al. Encorafenib plus binimetinib versus vemurafenib or encorafenib in patients with BRAF-mutant melanoma (COLUMBUS): a multicentre, openlabel, randomised phase 3 trial. Lancet Oncol. 2018;19:603-15.

12 Sarny S, Neumayer M, Kofler J, El-Shabrawi Y. Ocular toxicity due to trametinib and dabrafenib. BMC Ophthalmol. 2017;17(1):146.

13 Chan S, Ahern E, Chaudhry S, Hughes B. Bilateral acute anterior uveitis: a rare ocular side effect of erlotinib. BMJ Case Rep. 2019;12:e232868.

14 Joshi L, Karydis A, Gemenetzi M, Shao EH, Taylor SR. Uveitis as a result of MAP Kinase pathway inhibition. Case Rep Ophthalmol. 2013;4(3):279-82. 